\title{
Multiple bifurcations and periodic coexistence in a delayed Hopfield two-neural system with a monotonic activation function
}

\author{
Zigen Song ${ }^{1}$, Weiguo Qian², Bin Zhen ${ }^{3}$ and Xianghong Kong ${ }^{1 *}$
}

\section{"Correspondence:}

xhkong@shou.edu.cn

'College of Information Technology,

Shanghai Ocean University,

Shanghai, China

Full list of author information is

available at the end of the article

\section{Springer}

\begin{abstract}
In this paper, we consider a delayed Hopfield two-neural system with a monotonic activation function and find the periodic coexistence by bifurcation analysis. Firstly, we obtain the pitchfork bifurcation of the trivial equilibrium employing the central manifold and normal form methods. The neural system exhibits two pitchfork bifurcations near the trivial equilibrium. Then, analyzing the characteristic equation of the nontrivial equilibrium, we illustrate the saddle-node bifurcation of the nontrivial equilibria. The system exhibits the multi-coexistences of the stable and unstable equilibria. Further, we illustrate the plane regions of parameters having different numbers of equilibria. To obtain a time delay in neural system dynamics, we present the stability analysis and find the periodic orbit. The system exhibits stability switching by the Hopf bifurcation curves. Finally, the dynamic behaviors near the Hopf-Hopf bifurcation point are presented. The system exhibits coexistence of multiple periodic orbits with different frequencies.
\end{abstract}

Keywords: Hopfield neural system; Multiple delays; Coexistence; Multiple bifurcations; Monotonic activation function

\section{Introduction}

Hopfield neural network was firstly proposed by Hopfield in 1984 [1]. From then on, the neural network systems have seen great development, both regarding their properties and applications, such as in pattern recognition, signal processing, and associative memory [2, $3]$. The dynamical property of a neural system received much attention, which focuses on two main directions: establishing the stability conditions for equilibrium/periodic orbit [4-6] and discovering the complex dynamical behaviors, such as quasi-periodic orbit [7], chaos oscillation [8], and their coexistence $[9,10]$.

Bifurcation analysis is one of the most important tools to investigate the dynamic behaviors of a neural system. A bifurcation leads to a sudden topological change of system behaviors [11]. When a neural system is used as an associated memory, the stable equilibria of the neural network correspond to the static retrievable memory. So far, many scholars devoted their efforts to establish a neural network with coexisting multiple equilibria [12-14], which denotes the storage capacity of a neural system $[15,16]$. For the low-dimensional Hopfield neural network system, the existing dynamical analysis is focused on the local stability and Hopf bifurcation of the trivial equilibrium [17-19]. Re-

(c) The Author(s) 2019. This article is distributed under the terms of the Creative Commons Attribution 4.0 International License (http://creativecommons.org/licenses/by/4.0/), which permits unrestricted use, distribution, and reproduction in any medium, provided you give appropriate credit to the original author(s) and the source, provide a link to the Creative Commons license, and indicate if changes were made. 
cently, some codimension-two bifurcations, such as Bogdanov-Takens bifurcation [20, 21], pitchfork-Hopf bifurcation [22, 23], and Hopf-Hopf bifurcation [24, 25], were applied to investigate the neural system dynamics. In fact, it follows from the bifurcation theory that codimension-two bifurcations may induce different coexistence near the bifurcation points. For example, the Bogdanov-Takens and pitchfork-Hopf bifurcations will lead the system dynamics to the coexistence of an equilibrium and periodic orbit [26]. The Hopf-Hopf bifurcation induces the multi-coexistence of periodic orbits.

Due to the complexity of global dynamics for all trajectories [27], there is very little research on multiple equilibria and their stability in low-dimensional nonlinear systems. In fact, the low-dimensional Hopfield neural system may exhibit the multi-coexistence of equilibria and periodic orbits [28, 29]. Recently, Song et al. [30] employed the multistage pitchfork bifurcations of trivial and nontrivial equilibrium to find the multiple coexistences of stable and unstable equilibria in the Wilson-Cowan coupled system, which is a global dynamical analysis. When the activation function is defined as a non-monotonically increasing function, the neural system illustrates the multiple equilibria [31]. Ma and Wu [32] obtained a multi-coexistence of stable equilibrium in a two-dimensional neural system with the Morita activation function [33], which is a non-monotonically increasing function. However, to the best of our knowledge, there is no published report on the coexistence of multiple equilibria in the two-dimensional Hopfield neural system, and this motivates our present research. In this paper, the considered Hopfield neural system model is described by the following equations:

$$
\left\{\begin{array}{l}
\dot{x}_{1}(t)=-x_{1}(t)+\gamma f\left(x_{1}\left(t-\tau_{\mathrm{s}}\right)\right)+a_{21} f\left(x_{2}\left(t-\tau_{1}\right)\right), \\
\dot{x}_{2}(t)=-x_{2}(t)+a_{12} f\left(x_{1}\left(t-\tau_{1}\right)\right)+\gamma f\left(x_{2}\left(t-\tau_{\mathrm{s}}\right)\right),
\end{array}\right.
$$

where $x_{1}(t)$ and $x_{2}(t)$ describe the activities of neural populations at time $t, \gamma$ denotes the self-connection weights of the neural populations, $a_{12}$ and $a_{21}$ are the cross-interaction weights, $\tau_{1}>0$ and $\tau_{\mathrm{s}}>0$ are the coupled delays of cross- and self-interaction, the neural activation function is considered to be the hyperbolic tangent function $f(x)=\tanh (x)$, which is a monotonic function.

The rest of the paper is organized as follows. In the next section, the pitchfork bifurcations of the trivial equilibrium are analyzed employing the central manifold and normal form. The Hopfield neural network system exhibits two pitchfork bifurcations near the trivial equilibrium. In Sect. 3, the static bifurcation of the nontrivial equilibrium is investigated. The system has two saddle-node bifurcations of the nontrivial equilibria. It implies that the system exhibits multiple coexistence of stable equilibria. By the bifurcation curves, we give the detailed regions having different numbers of the system equilibria. In Sect. 4, we will exhibit the stability analysis of the trivial equilibrium and find the periodic orbit using the Hopf bifurcation. In Sect. 5, we will find the stability regions to illustrate the stability switching by the Hopf bifurcation curves. Further, some Hopf-Hopf bifurcation points are presented, which are the intersection points of the Hopf bifurcation curves. The dynamic behaviors near these bifurcation points are illustrated. The system exhibits periodic orbits with different frequencies and the periodic coexistence in the different parameter regions near the bifurcation points. Conclusions and discussions are given in Sect. 6. 


\section{Pitchfork bifurcation of the trivial equilibrium}

It is obvious that system (1.1) has the trivial equilibrium $\left(x_{1}, x_{2}\right)=(0,0)$. By letting $\dot{x}_{1}=0$, $\dot{x}_{2}=0$ in system (1.1), we obtain that the nontrivial equilibrium $\left(x_{10}, x_{20}\right)$ must fulfill $x_{10}=$ $\gamma f\left(x_{10}\right)+a_{21} f\left(x_{20}\right)$ and $x_{20}=a_{12} f\left(x_{10}\right)+\gamma f\left(x_{20}\right)$, which are independent of time delays $\tau_{1}$ and $\tau_{\mathrm{s}}$. In this section, we will describe the evolution process of the equilibria number employing pitchfork bifurcation (a type of static bifurcation) of the trivial equilibrium. Since time delay has no-impact on the number of system equilibria, we rewrite system (1.1) as the following non-delayed system (i.e., $\tau_{1}=0$ and $\tau_{\mathrm{s}}=0$ ), which is

$$
\left\{\begin{array}{l}
\dot{x}_{1}(t)=-x_{1}(t)+\gamma f\left(x_{1}(t)\right)+a_{21} f\left(x_{2}(t)\right), \\
\dot{x}_{2}(t)=-x_{2}(t)+a_{12} f\left(x_{1}(t)\right)+\gamma f\left(x_{2}(t)\right) .
\end{array}\right.
$$

The linearization of the system at the trivial equilibrium $(0,0)$ produces

$$
\left\{\begin{array}{l}
\dot{x}_{1}(t)=-x_{1}(t)+\gamma x_{1}(t)+a_{21} x_{2}(t), \\
\dot{x}_{2}(t)=-x_{2}(t)+a_{12} x_{1}(t)+\gamma x_{2}(t) .
\end{array}\right.
$$

The associated characteristic equation is

$$
\lambda^{2}+(2-2 \gamma) \lambda+\gamma^{2}-2 \gamma-a_{12} a_{21}+1=0 .
$$

System (2.1) undergoes a static bifurcation at the trivial equilibrium when the system eigenvalue traverses the imaginary axis along the real axis. Letting $\lambda=0$ in (2.3), one has

$$
\gamma^{2}-2 \gamma-a_{12} a_{21}+1=0
$$

That is,

$$
\gamma_{1}=1-\sqrt{a_{12} a_{21}} \text { and } \gamma_{2}=1+\sqrt{a_{12} a_{21}}
$$

where $a_{12} a_{21}>0$. It is a static bifurcation point of the trivial equilibrium. In what follows, we will exhibit the corresponding normal form to show the bifurcation types (i.e., saddlenode, pitchfork, or transcritical bifurcation) by the central manifold and normal form. The result shows that the static bifurcations are all pitchfork bifurcations. This implies that system (2.1) undergoes multiple pitchfork bifurcations at the trivial equilibrium. To this end, letting $\gamma=\gamma_{1}+\varepsilon$, where $\varepsilon$ is the unfolding parameter of the bifurcation parameter, we have the following theorem.

Theorem 1 System (2.1) is reduced to the following normal form system near the bifurcation point $\gamma=\gamma_{1}$, which implies that system (2.1) undergoes a pitchfork bifurcation at the trivial equilibrium with $a_{21} \neq a_{12}$. Moreover, the pitchfork bifurcation is supercritical for $a_{12} a_{21}-a_{12}^{2}<0$, and subcritical for $a_{12} a_{21}-a_{12}^{2}>0$. The normal form is

$$
\left\{\begin{array}{l}
\dot{z}_{1}=\varepsilon z_{1}+\left(a_{21}-a_{12}\right) z_{1}^{3} / 6 a_{12}+\cdots \\
\dot{\varepsilon}=0
\end{array}\right.
$$


Proof Let $\gamma=1-\sqrt{a_{12} a_{21}}$. The characteristic equation is simplified to

$$
P(\lambda)=\left(2 \sqrt{a_{12} a_{21}}+\lambda\right) \lambda=0 .
$$

The eigenvalues are

$$
\lambda_{1}=0, \quad \lambda_{2}=-2 \sqrt{a_{12} a_{21}} .
$$

The corresponding eigenvectors are

$$
v_{1}=\left(\begin{array}{c}
\sqrt{a_{21} / a_{12}} \\
1
\end{array}\right), \quad v_{2}=\left(\begin{array}{c}
-\sqrt{a_{21} / a_{12}} \\
1
\end{array}\right) .
$$

Substitute $\gamma=\gamma_{1}+\varepsilon$ into (2.1), where $\varepsilon$ is the unfolding parameter. Using Taylor expansion, one has the following extended system:

$$
\left\{\begin{array}{l}
\dot{x}_{1}=\left(\gamma_{1}+\varepsilon-1\right) x_{1}+a_{21} x_{2}-\left(\gamma_{1}+\varepsilon\right) x_{1}^{3} / 3-a_{21} x_{2}^{3} / 3+\cdots \\
\dot{x}_{2}=a_{12} x_{1}+\left(\gamma_{1}+\varepsilon-1\right) x_{2}-a_{12} x_{1}^{3} / 3-\left(\gamma_{1}+\varepsilon\right) x_{2}^{3} / 3+\cdots \\
\dot{\varepsilon}=0
\end{array}\right.
$$

Let

$$
\left(\begin{array}{l}
x_{1} \\
x_{2}
\end{array}\right)=\mathrm{T}\left(\begin{array}{l}
z_{1} \\
z_{2}
\end{array}\right), \quad \text { where } \mathrm{T}=\left(\begin{array}{cc}
\sqrt{a_{21} / a_{12}} & -\sqrt{a_{21} / a_{12}} \\
1 & 1
\end{array}\right)
$$

System (2.9) can be transformed into the following standard form:

$$
\left\{\begin{array}{l}
\dot{z}_{1}=0 z_{1}+\varepsilon z_{1}+q_{1} z_{1}^{3}+3 q_{1} z_{1}^{2} z_{2}+3 q_{1} z_{1} z_{2}^{2}+q_{1} z_{2}^{3}+\cdots \\
\dot{z}_{2}=\lambda_{2} z_{2}+\varepsilon z_{2}+q_{2} z_{1}^{3}+q_{3} z_{1}^{2} z_{2}+q_{2} z_{1} z_{2}^{2}+q_{3} z_{2}^{3}+\cdots \\
\dot{\varepsilon}=0
\end{array}\right.
$$

where

$$
\begin{aligned}
& q_{1}=\left(a_{21}-a_{12}\right) / 6 a_{12}, q_{2}=\left(a_{21}-a_{12}+2 a_{12} \sqrt{a_{12} a_{21}}-2 a_{21} \sqrt{a_{12} a_{21}}\right) / 6 a_{12}, \\
& q_{3}=\left(a_{21}-a_{12}-2 a_{12} \sqrt{a_{12} a_{21}}-2 a_{21} \sqrt{a_{12} a_{21}}\right) / 6 a_{12} .
\end{aligned}
$$

To reduce system (2.10) onto the center manifold, we assume the central manifold as

$$
W^{c}(0)=\left\{\left(z_{1}, z_{2}, \varepsilon\right) \in R^{3}\left|z_{2}=h_{2}\left(z_{1}, \varepsilon\right),\right| z_{1}\left|<\delta_{1},\right| \varepsilon \mid<\delta_{2}, h_{2}(0,0)=0, D h_{2}(0,0)=0\right\},
$$

where

$$
z_{2}=h_{2}\left(z_{1}, \varepsilon\right)=r_{1} z_{1}^{2}+r_{2} z_{1} \varepsilon+r_{3} \varepsilon^{2}+\cdots
$$


and $\delta_{1}$ and $\delta_{2}$ are sufficiently small. According to the center manifold theorem, the center manifold must satisfy

$$
\mathrm{D} h_{2}\left(0 z_{1}+f_{1}\right)-\lambda_{2} h_{2}-f_{2}=0
$$

where $\mathrm{D}$ is the differential operator, and

$$
\left\{\begin{array}{l}
f_{1}=\varepsilon z_{1}+q_{1} z_{1}^{3}+3 q_{1} z_{1}^{2} z_{2}+3 q_{1} z_{1} z_{2}^{2}+q_{1} z_{2}^{3}+\cdots \\
f_{2}=\varepsilon z_{2}+q_{2} z_{1}^{3}+q_{3} z_{1}^{2} z_{2}+q_{2} z_{1} z_{2}^{2}+q_{3} z_{2}^{3}+\cdots
\end{array}\right.
$$

Substituting (2.11) into (2.12) and setting the coefficients of the same powers to zero, the coefficients of Eq. (2.11) can be obtained. So the vector field reduced into the center manifold is exhibited as system (2.6). This completes the proof.

Using the same computational process, we obtain the following theorem for the second bifurcation point $\gamma=\gamma_{2}$.

Theorem 2 System (2.1) can be reduced to the following normal form system near the bifurcation point $\gamma=\gamma_{2}$, which implies that system (2.1) undergoes a pitchfork bifurcation at the trivial equilibrium with $a_{21} \neq-a_{12}$. Moreover, the pitchfork bifurcation is supercritical because of $a_{12} a_{21}+a_{12}^{2}>0$. The normal form is

$$
\left\{\begin{array}{l}
\dot{z}_{1}=\varepsilon z_{1}-\left(a_{12}+a_{21}\right) z_{1}^{3} / 6 a_{12}+\cdots \\
\dot{\varepsilon}=0
\end{array}\right.
$$

It follows from Theorems 1 and 2 that system (2.1) has two pitchfork bifurcations $\gamma_{1}=1-\sqrt{a_{12} a_{21}}$ and $\gamma_{2}=1+\sqrt{a_{12} a_{21}}$ when $a_{12} a_{21}>0$ is satisfied. That is to say, system (2.1) exhibits multiple nontrivial equilibria employing the pitchfork bifurcation of the trivial equilibrium. For example, choosing the system parameters as $a_{12}=1, a_{21}=0.6$, one obtains the pitchfork bifurcations $\gamma_{1}=0.2254$ and $\gamma_{2}=1.7746$. Further, it follows from Theorems 1 and 2 that $a_{12} a_{21}-a_{12}^{2}=-0.4<0$ and $a_{12} a_{21}+a_{12}^{2}=1.6>0$, which implies that the bifurcations are all supercritical. In fact, system (2.1) has the dynamic nullclines $x_{1}=\gamma f\left(x_{1}\right)+a_{21} f\left(x_{2}\right)$ and $x_{2}=a_{12} f\left(x_{1}\right)+\gamma f\left(x_{2}\right)$, which are shown in Fig. 1(a) for the fixed value $\gamma=0.1$. There is a single trivial equilibrium. System (1.1) exhibits the first supercritical pitchfork bifurcation of the trivial equilibrium when $\gamma=0.2254$. The single trivial equilibrium evolves into three equilibria, one is the unstable trivial equilibrium and the others are the stable nontrivial equilibria, as shown in Fig. 1(b) for the fixed value $\gamma=0.5$. Further increasing the system parameter $\gamma$, we reach and cross the critical value $\gamma_{2}=1.7746$. System (2.1) exhibits the second pitchfork bifurcation of the trivial equilibrium, as shown in Figs. 1(c) and 1(d). This implies that the unstable trivial equilibrium bifurcates into two unstable equilibria. At this time, system (2.1) exhibits one unstable trivial equilibrium, two stable equilibria, and two unstable equilibria.

\section{Saddle-node bifurcation of the nontrivial equilibrium}

In the above-mentioned section, we obtained the multiple pitchfork bifurcations of the trivial equilibrium employing the central manifold and normal form. System (2.1) presented multiple nontrivial equilibria. In what follows, we discuss the static bifurcation of 


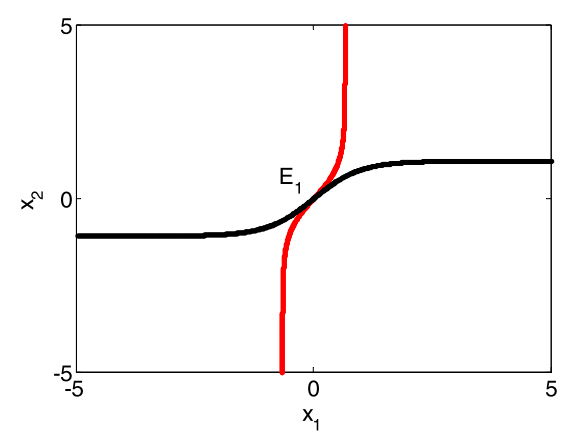

(a)

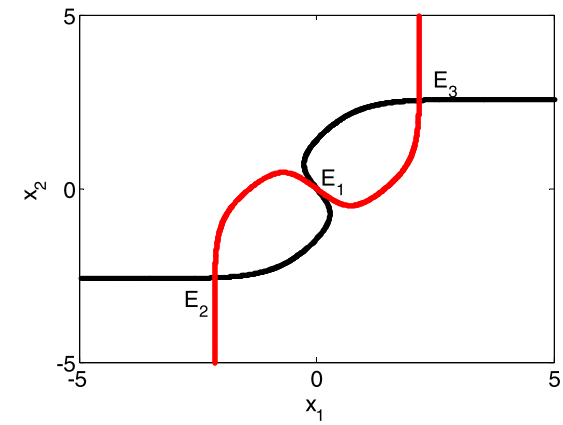

(c)

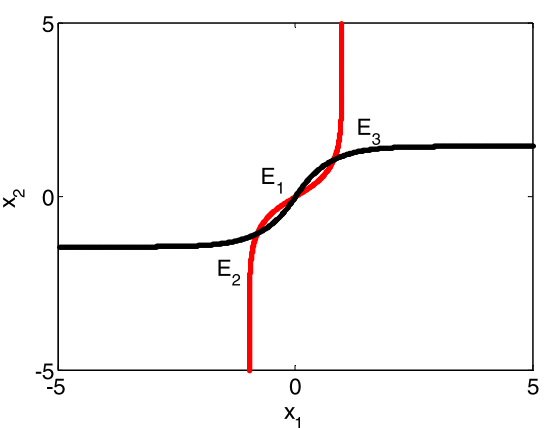

(b)

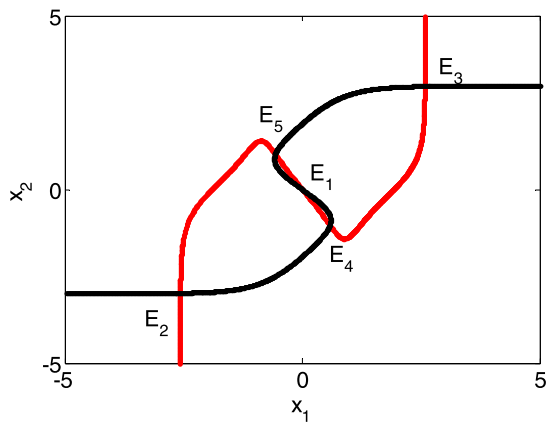

(d)

Figure 1 System nullclines illustrate the equilibrium numbers with (a) $\gamma=0.1$, (b) $\gamma=0.5$, (c) $\gamma=1.6$, and (d) $\gamma=2.0$ for the given parameter values $a_{12}=1, a_{21}=0.6$

the nontrivial equilibrium generated by the pitchfork bifurcation. To this end, suppose $\left(x_{10}, x_{20}\right)$ is a nontrivial equilibrium of system (2.1). By transforming $u_{1}(t)=x_{1}(t)-x_{10}$, $u_{2}(t)=x_{2}(t)-x_{20}$, we have the corresponding linearization system

$$
\left\{\begin{array}{l}
\dot{u}_{1}(t)=-u_{1}(t)+\gamma M u_{1}(t)+a_{21} N u_{2}(t) \\
\dot{u}_{2}(t)=-u_{2}(t)+a_{12} M u_{1}(t)+\gamma N u_{2}(t)
\end{array}\right.
$$

where $M=1-\tanh ^{2}\left(x_{10}\right), N=1-\tanh ^{2}\left(x_{20}\right)$. The corresponding characteristic equation is

$$
\left|\begin{array}{cc}
\lambda+1-\gamma M & -a_{21} N \\
-a_{12} M & \lambda+1-\gamma N
\end{array}\right|=0
$$

which is equivalent to

$$
1-\gamma M-\gamma N+\gamma^{2} M N-a_{12} a_{21} M N+(2-\gamma M-\gamma N) \lambda+\lambda^{2}=0 .
$$

It follows from the bifurcation theory that system (2.1) has a static bifurcation at the nontrivial equilibrium $\left(x_{10}, x_{20}\right)$ if the following equation is valid:

$$
L(\gamma)=1-\gamma M-\gamma N+\gamma^{2} M N-a_{12} a_{21} M N=0 .
$$


Figure 2 The parameter curves of $L(\gamma)$ illustrate the static bifurcation point for the trivial equilibria (blue) and nontrivial equilibrium (red) for the fixed parameters $a_{12}=1, a_{21}=0.6$

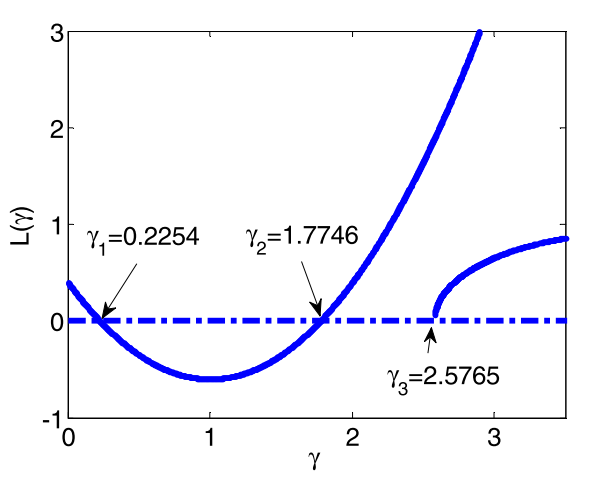

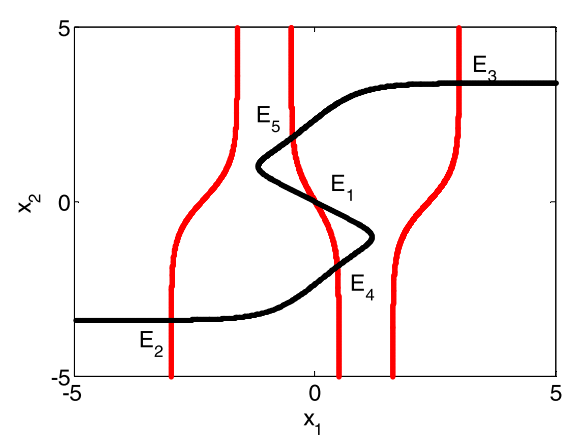

(a)

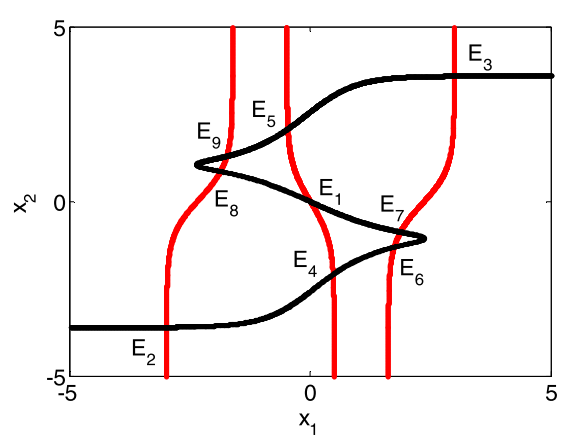

(b)

Figure 3 Intersection points of the nullclines illustrate the saddle-node bifurcation of the nontrivial equilibrium: (a) five equilibria for $\gamma=2.4$ and (b) nine equilibria for $\gamma=2.6$. The parameter values are given by $a_{12}=1, a_{21}=0.6$

It should be noted that $M$ and $N$ depend on the system parameters $\gamma, a_{12}$, and $a_{21}$. So Eq. (3.4) is a transcendental and complicated equation. The corresponding static bifurcation point of the nontrivial equilibrium cannot be illustrated in a theoretical expression. But for the given parameters, the bifurcation point can be obtained by some numerical computations.

For example, as in the previous section, the system parameters are fixed as $a_{12}=1$, $a_{21}=0.6$. There are five equilibria when $\gamma$ crosses the second pitchfork bifurcation, i.e., $\gamma>\gamma_{2}=1.774$. Figure 2 shows the parameter curves of $L(\gamma)$ given by (3.4). It follows that $L(\gamma)=0$ has three roots $\gamma_{1}=0.2254, \gamma_{2}=1.7746$, and $\gamma_{3}=2.5765$, where $\gamma_{1}=0.2254$ and $\gamma_{2}=1.7746$ are the static bifurcations of the trivial equilibrium. The third root of Eq. (3.4) is the bifurcation point of the nontrivial equilibrium, that is, $\gamma_{3}=2.5765$. To illustrate the bifurcation type of the nontrivial equilibrium, we exhibit the dynamic nullclines $x_{1}=\gamma f\left(x_{1}\right)+a_{21} f\left(x_{2}\right)$ and $x_{2}=a_{12} f\left(x_{1}\right)+\gamma f\left(x_{2}\right)$ for the parameter values near the bifurcation point $\gamma_{3}=2.5765$. It follows from Fig. 3 that the dynamic nullclines just have five intersection points for $\gamma=2.4$, which is generated by the multiple pitchfork bifurcation of the trivial equilibrium, as shown in Fig. 3(a). However, there are nine intersection points for $\gamma=2.6$, as shown in Fig. 3(b). This implies that system (2.1) exhibits simultaneously two saddle-node bifurcation of the nontrivial equilibrium. Two pairs of stable and unstable nontrivial equilibria will be generated. The result shows that system (2.1) exhibits nine equilibria for $\gamma>\gamma_{3}$, namely, one unstable trivial equilibrium, two unstable nontrivial 
Figure 4 The one-dimensional bifurcation diagrams for the trivial equilibrium for the fixed system parameters $a_{12}=1, a_{21}=0.6$

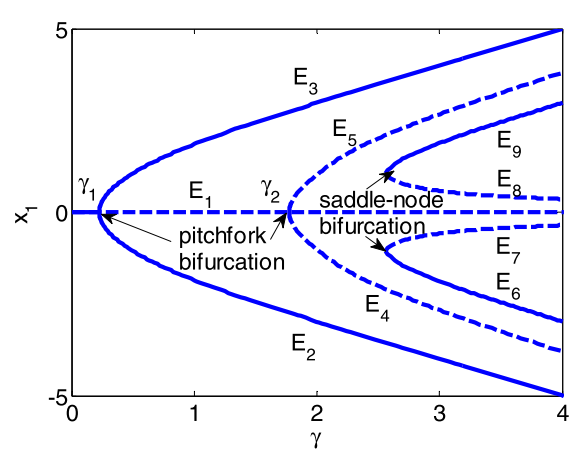

Figure 5 The multistage static bifurcation curves divide the parameter $\left(\gamma, a_{21}\right)$-plane into the different regions having the different numbers of equilibria for $a_{12}=1$

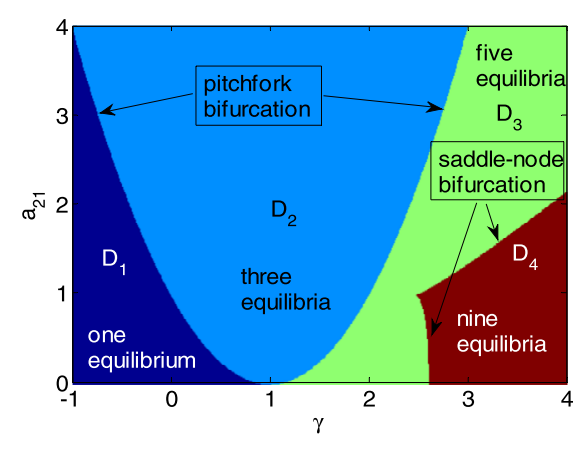

equilibria, and two stable nontrivial equilibria. The one-dimensional bifurcation diagram with varying $\gamma$ is exhibited in Fig. 4.

The parameter $\left(\gamma, a_{21}\right)$-plane is divided into several different regions having different numbers of system equilibria by the static bifurcation curves of trivial and nontrivial equilibria, as shown in Fig. 5 for $a_{12}=1>0$. It should be noticed that similar results can be obtained by numerical simulation for $a_{12}=-1<0$. The system has one, three, five, and nine equilibria for the different regions of system parameters. In region $\mathrm{D}_{1}$, there is a stable trivial equilibrium shown as Fig. 1(a). If the system parameter passes through the first pitchfork bifurcation curve $\gamma=1-\sqrt{a_{12} a_{21}}$ and enters into region $\mathrm{D}_{2}$, system (2.1) has three equilibria (one unstable trivial equilibrium and a pair of stable nontrivial equilibria, as shown in Figs. 1(b) and 1(c)) by the pitchfork bifurcation of the trivial equilibrium. Further, the second pitchfork bifurcation curve of the trivial equilibrium is the region boundary of three and five equilibria. When the system parameter passes through the curve $\gamma=1+\sqrt{a_{12} a_{21}}$ and enters into region $\mathrm{D}_{3}$, a new pair of nontrivial equilibria will be exhibited. System (2.1) presents five equilibria with one unstable trivial, a pair of stable and a pair of unstable nontrivial equilibria, as shown in Figs. $1(\mathrm{~d})$ and $3(\mathrm{a})$. In region $\mathrm{D}_{4}$, two pairs of new nontrivial equilibria are presented when the parameter passes through the saddle-node bifurcation of the nontrivial equilibrium. It implies that system (2.1) has nine equilibria, namely, one unstable trivial equilibrium, two unstable nontrivial equilibria, and four stable nontrivial equilibria, as shown in Fig. 3(b).

\section{Stability analysis and Hopf bifurcation}

In this section, we will exhibit the stability analysis of the trivial equilibrium and find the periodic orbit using the Hopf bifurcation theory. To this end, we firstly obtain the lineariz- 
ing system at the trivial equilibrium, which is

$$
\left\{\begin{array}{l}
\dot{x}_{1}(t)=-x_{1}(t)+\gamma x_{1}\left(t-\tau_{\mathrm{s}}\right)+a_{21} x_{2}\left(t-\tau_{1}\right), \\
\dot{x}_{2}(t)=-x_{2}(t)+a_{12} x_{1}\left(t-\tau_{1}\right)+\gamma x_{2}\left(t-\tau_{\mathrm{s}}\right) .
\end{array}\right.
$$

The characteristic equation of system (4.1) is

$$
1-2 \gamma \mathrm{e}^{-\lambda \tau_{\mathrm{s}}}+\gamma^{2} \mathrm{e}^{-2 \lambda \tau_{\mathrm{s}}}-a_{12} a_{21} \mathrm{e}^{-2 \lambda \tau_{1}}+\left(2-2 \gamma \mathrm{e}^{-\lambda \tau_{\mathrm{s}}}\right) \lambda+\lambda^{2}=0 .
$$

System (1.1) has a stable equilibrium when all the eigenvalues of Eq. (4.2) have negative real parts. To simplify, we show the stability conditions for system (1.1) with $\tau_{\mathrm{s}}=0$, first. That is,

$$
1-2 \gamma+\gamma^{2}-a_{12} a_{21} \mathrm{e}^{-2 \lambda \tau_{1}}+(2-2 \gamma) \lambda+\lambda^{2}=0 .
$$

Supposing $\tau_{1}=0$ in Eq. (4.3) produces

$$
1-2 \gamma+\gamma^{2}-a_{12} a_{21}+(2-2 \gamma) \lambda+\lambda^{2}=0 .
$$

By using the Routh-Hurwitz criterion, we obtain the basic (necessary and sufficient) condition to assure that the trivial equilibrium of system (1.1) has local stability for $\tau_{1}=0$ and $\tau_{\mathrm{s}}=0$, which is

$$
\gamma<1, \quad a_{12} a_{21}<(\gamma-1)^{2} .
$$

If delay $\tau_{1}$ increases, the trivial equilibrium may lose its stability and evolve into an unstable equilibrium. To obtain the critical values, letting Eq. (4.3) have the pure imaginary roots $\lambda= \pm i v, v>0$, one has

$$
1-2 \gamma+\gamma^{2}-a_{12} a_{21} \mathrm{e}^{-2 i v \tau_{1}}+(2-2 \gamma) i v-v^{2}=0 .
$$

Separating Eq. (4.6) into real and imaginary parts produces

$$
\left\{\begin{array}{l}
1-v^{2}-2 \gamma+\gamma^{2}-a_{12} a_{21} \cos 2 v \tau_{1}=0 \\
2 v-2 v \gamma+a_{12} a_{21} \sin 2 v \tau_{1}=0
\end{array}\right.
$$

Eliminating $\tau_{1}$ from Eq. (4.7) by $\sin ^{2} 2 v \tau_{1}+\cos ^{2} 2 v \tau_{1}=1$, one has

$$
L(v)=\left(v^{2}+(\gamma-1)^{2}\right)^{2}-\left(a_{12} a_{21}\right)^{2}=0 .
$$

Based on the basic conditions (4.5), we have the root of Eq. (4.8) if $a_{12} a_{21}<-(\gamma-1)^{2}$, which is

$$
v=\sqrt{-a_{12} a_{21}-(\gamma-1)^{2}} .
$$




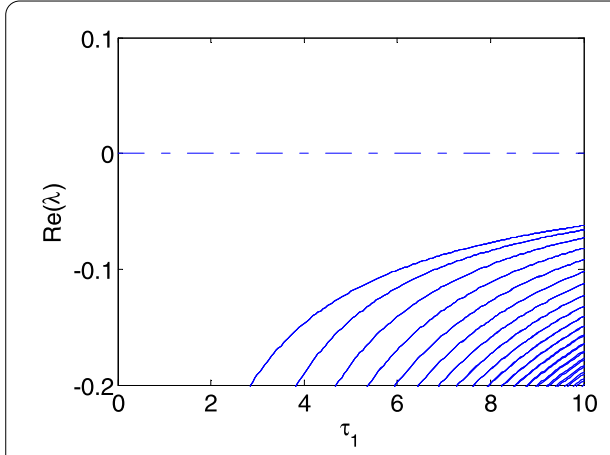

(a)

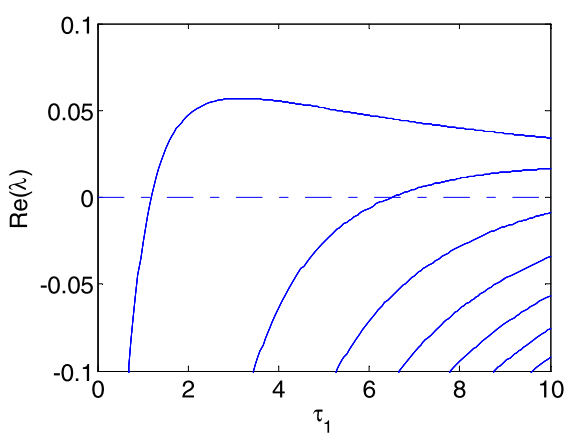

(b)

Figure 6 The real parts of system eigenvalues with $\tau_{1}$ increasing: (a) $\gamma=-0.5$ and (b) $\gamma=0.5$. The other parameters are $a_{12}=1, a_{21}=-0.6$, and $\tau_{\mathrm{s}}=0$

Then Eq. (4.7) has the minimal value of the critical delay $\tau_{1}^{0}=\varphi / 2 \nu$, where $\varphi \in[0,2 \pi)$ satisfies

$$
\left\{\begin{array}{l}
1-v^{2}-2 \gamma+\gamma^{2}-a_{12} a_{21} \cos \varphi=0 \\
2 v-2 v \gamma+a_{12} a_{21} \sin \varphi=0
\end{array}\right.
$$

This implies that all the eigenvalues of Eq. (4.6) have negative real parts for any delay $\tau_{1}$ when $\gamma<1,\left|a_{12} a_{21}\right|<(\gamma-1)^{2}$. The trivial equilibrium of system (1.1) presents the alldelayed stability. However, when $\gamma<1, a_{12} a_{21}<-(\gamma-1)^{2}$, there exists a critical value $\tau_{1}^{0}=$ $\varphi / 2 v$, where all the eigenvalues of Eq. (4.6) have negative real parts for the delay interval $\tau_{1} \in\left(0, \tau_{1}^{0}\right)$ and the least root with a positive part for $\tau_{1} \in\left(\tau_{1}^{0},+\infty\right)$. The trivial equilibrium will change the local dynamics from stable to instable, which is called the delay-dependent stability. The examples are illustrated in Fig. 6. It follows from Fig. 6(a) that all the eigenvalues have negative real parts for $\gamma=-0.5<1$, where $0.6=\left|a_{12} a_{21}\right|<(\gamma-1)^{2}=2.25$. However, when we choose $\gamma=0.5<1$, then $-0.6=a_{12} a_{21}<-(\gamma-1)^{2}=-0.025$. It follows from Eq. (4.9) that $v=0.5916$. There is a critical value of the delay $\tau_{1}^{0}=\varphi / 2 v$. All the eigenvalues of Eq. (4.6) have negative real parts for the delay interval $\tau_{1} \in\left(0, \tau_{1}^{0}\right)$ and the least root with a positive part for $\tau_{1} \in\left(\tau_{1}^{0},+\infty\right)$.

Further, to analyze the effect of $\tau_{1}$ and $\tau_{\mathrm{s}}$ on the stability of the trivial equilibrium in system (1.1), we regard $\tau_{\mathrm{s}}$ as the variable parameter for the chosen delay $\tau_{1}$. Supposing $\lambda=i \omega, \omega>0$ is the root of the characteristic equation (4.2), we have

$$
1-2 \gamma \mathrm{e}^{-i \omega \tau_{\mathrm{s}}}+\gamma^{2} \mathrm{e}^{-2 i \omega \tau_{\mathrm{s}}}-a_{12} a_{21} \mathrm{e}^{-2 i \omega \tau_{1}}+\left(2-2 \gamma \mathrm{e}^{-i \omega \tau_{\mathrm{s}}}\right) i \omega-\omega^{2}=0 .
$$

Multiplying by $\mathrm{e}^{i \omega \tau_{\mathrm{s}}}$ in (4.10) and separating real and imaginary parts produces

$$
\left\{\begin{array}{l}
-2 \gamma+\left(1+\gamma^{2}-\omega^{2}\right) \cos \omega \tau_{\mathrm{s}}-a_{12} a_{21} \cos \left(2 \omega \tau_{1}-\omega \tau_{\mathrm{s}}\right)-2 \omega \sin \omega \tau_{\mathrm{s}}=0 \\
-2 \gamma \omega+2 \omega \cos \omega \tau_{\mathrm{s}}+a_{12} a_{21} \cos \left(2 \omega \tau_{1}-\omega \tau_{\mathrm{s}}\right)+\left(1-\gamma^{2}-\omega^{2}\right) \sin \omega \tau_{\mathrm{s}}=0
\end{array}\right.
$$

Eliminating $\tau_{\mathrm{s}}$ from (4.11), we have

$$
\cos \omega \tau_{\mathrm{s}}=k_{1}(\omega) / k_{3}(\omega), \sin \omega \tau_{\mathrm{s}}=k_{2}(\omega) / k_{3}(\omega),
$$


where

$$
\begin{aligned}
& k_{1}(\omega)=2 \gamma\left(1-\gamma^{2}+\omega^{2}-a_{12} a_{21}\left(\cos 2 \omega \tau_{1}-\omega \sin 2 \omega \tau_{1}\right),\right. \\
& k_{2}(\omega)=2 \gamma\left(\gamma^{2} \omega-\omega-\omega^{3}-a_{12} a_{21}\left(\omega \cos 2 \omega \tau_{1}+\sin 2 \omega \tau_{1}\right),\right. \\
& k_{3}(\omega)=a_{12}^{2} a_{21}^{2}-\gamma^{4}+\left(1+\omega^{2}\right)^{2}-2 a_{12} a_{21}\left(1-\omega^{2}\right) \cos 2 \omega \tau_{1}+4 a_{12} a_{21} \omega \sin 2 \omega \tau_{1} .
\end{aligned}
$$

Using $\cos ^{2} \omega \tau_{\mathrm{s}}+\sin ^{2} \omega \tau_{\mathrm{s}}=1$, we have

$$
K(\omega)=k_{1}^{2}(\omega)+k_{2}^{2}(\omega)-k_{3}^{2}(\omega)=0
$$

In general, assume Eq. (4.13) exhibits positive roots $\omega_{i}, i=1,2, \ldots$. Then Eq. (4.2) has critical values, namely,

$$
\tau_{\mathrm{s}}^{i, j}=\frac{\varphi_{i}+2 j \pi}{\omega_{i}}, \quad i=1,2, \ldots ; j=0,1,2, \ldots
$$

where $\varphi_{i} \in[0,2 \pi)$. Define

$$
\tau_{\mathrm{s}}^{0}=\min \left\{\tau_{\mathrm{s}}^{i, 0}: i=1,2, \ldots\right\}
$$

To ensure the Hopf bifurcation occurs, we find the transversality condition, i.e., the eigenvalue of Eq. (4.2) crosses the imaginary axis with a non-zero velocity. Differentiating $\lambda$ with respect to $\tau_{\mathrm{s}}$ in Eq. (4.2), we have the crossing velocity

$$
\lambda^{\prime}\left(\tau_{\mathrm{s}}\right)=\frac{\gamma \mathrm{e}^{2 \lambda \tau_{1}} \lambda\left(\gamma-\mathrm{e}^{\lambda \tau_{\mathrm{s}}}(1+\lambda)\right)}{\mathrm{e}^{2 \lambda\left(\tau_{1}+\tau_{\mathrm{s}}\right)}(1+\lambda)+a_{12} a_{21} \mathrm{e}^{2 \lambda \tau_{\mathrm{s}}} \tau_{1}-\mathrm{e}^{2 \lambda \tau_{1}} \gamma^{2} \tau_{\mathrm{s}}+\mathrm{e}^{\lambda\left(2 \tau_{1}+\tau_{\mathrm{s}}\right)} \gamma\left(-1+\tau_{\mathrm{s}}+\lambda \tau_{\mathrm{s}}\right)} .
$$

Based on the Hopf bifurcation theory, the following conclusion can be obtained under the basic condition (4.5), i.e., $\gamma<1, a_{12} a_{21}<(\gamma-1)^{2}$. If there exists no positive root of Eq. (4.13), all the eigenvalues of Eq. (4.2) exhibit negative real parts for $\tau_{\mathrm{s}} \geq 0$. The trivial equilibrium has the all-delayed stability. Further, if $K(\omega)=0$ has at least one positive root among $\omega_{i}, i=1,2, \ldots$, there is a critical delay $\tau_{\mathrm{s}}^{0}$, where all the eigenvalues exhibit negative real parts for $\tau_{\mathrm{s}} \in\left(0, \tau_{\mathrm{s}}^{0}\right)$. The trivial equilibrium is asymptotically stable. Moreover, system (1.1) exhibits a Hopf bifurcation at the trivial equilibrium for $\tau_{\mathrm{s}}=\tau_{\mathrm{s}}^{0}$ if $\operatorname{Re}\left(\lambda^{\prime}\left(\tau_{\mathrm{s}}\right)\right) \neq 0$ is satisfied. A periodic orbit will be presented near the trivial equilibrium.

For example, if we choose the system parameters as $a_{12}=1, a_{21}=-0.6$, and $\gamma=0.5$, it follows from $a_{12} a_{21}=-0.6<1$ that system (1.1) has a single trivial equilibrium. Further, $K(\omega)=0$ has no positive root for $\tau_{1}=0.7$ and positive ones for $\tau_{1}=1.1$. Employing the above conclusion that the trivial equilibrium is all-delayed stable for $\tau_{1}=0.7$, the corresponding time histories are illustrated in Figs. 7 (a) and 7(b) with a small delay $\tau_{\mathrm{s}}=1$ and large delay $\tau_{\mathrm{s}}=9$. All trajectories evolve into the trivial equilibrium. However, when $\tau_{1}$ increases to $1.1, K(\omega)=0$ produces the positive roots. The minimum delay of the Hopf bifurcation is $\tau_{\mathrm{s}}^{0}=7.1957$ by using Eq. (4.14). It implies that the trivial equilibrium is locally stable for $\tau_{\mathrm{s}} \in(0,7.1957)$, as shown in Fig. 8(a) with the fixed delay $\tau_{\mathrm{s}}=1$. Further, system (1.1) undergoes a Hopf bifurcation when $\tau_{\mathrm{s}}$ goes through the critical value $\tau_{\mathrm{s}}^{0}=7.1957$. The system dynamics near the trivial equilibrium will lose its stability and enter a stable periodic orbit, as shown in Fig. 8(b) for the fixed delay $\tau_{\mathrm{s}}=9$. 


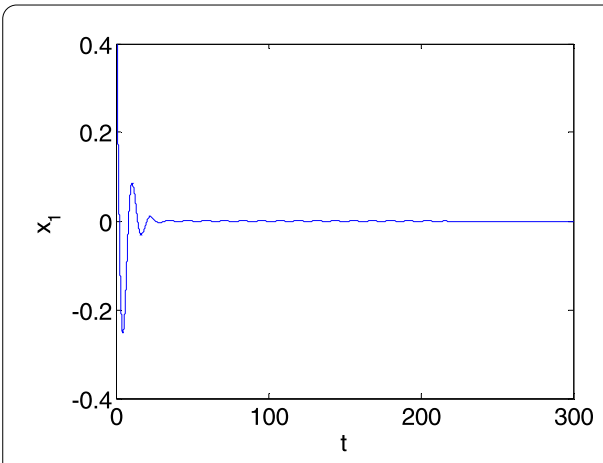

(a)

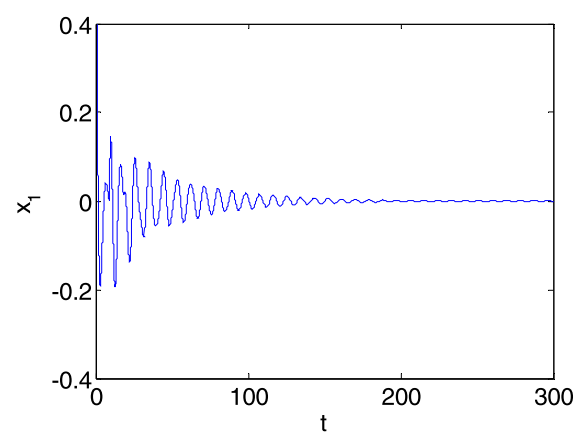

(b)

Figure 7 Time histories of system (1.1) with (a) $\tau_{\mathrm{s}}=1$ and (b) $\tau_{\mathrm{s}}=9$ for the fixed parameters $a_{12}=1$, $a_{21}=-0.6, \gamma=0.5$, and $\tau_{1}=0.7$

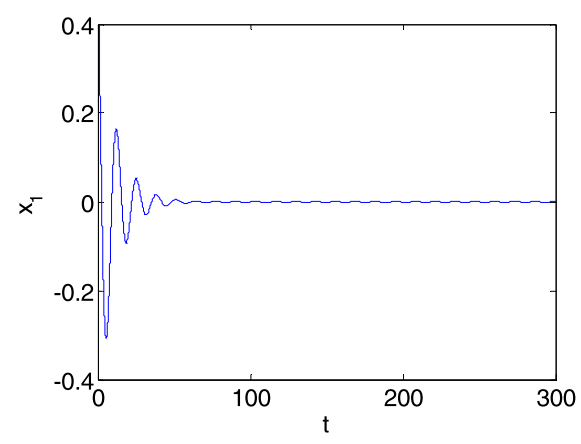

(a)

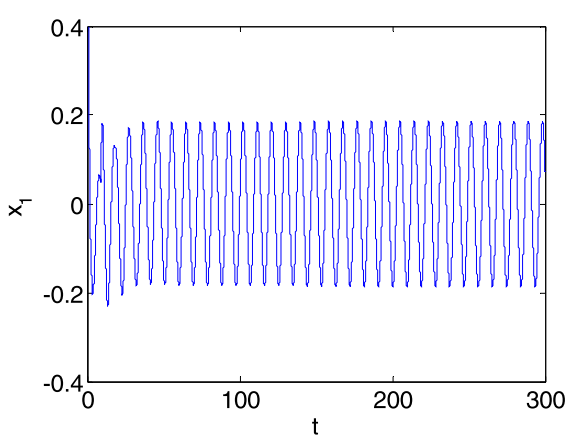

(b)

Figure 8 Time histories of system (1.1) with (a) $\tau_{\mathbf{s}}=1$ and (b) $\tau_{\mathbf{s}}=9$ for the fixed parameters $a_{12}=1$, $a_{21}=-0.6, \gamma=0.5$, and $\tau_{1}=1.1$

\section{Hopf-Hopf bifurcation and periodic coexistence}

It follows from the above section that system (1.1) produces the Hopf bifurcation at the trivial equilibrium if equation $K(\omega)=0$ has a positive root, which implies that the system dynamical behavior enters into a stable periodic orbit. In fact, the multiple roots of $K(\omega)=0$ will lead the system dynamics into stability switching. At this time, the system dynamics will lose and then regain its stability with the increasing delay. In this section, we will find the stability regions to illustrate the stability switching by the Hopf bifurcation curves. Further, some Hopf-Hopf bifurcation points will be presented by exhibiting the intersection points of the Hopf bifurcation curves. The dynamic behaviors near these bifurcation points will be shown. The system will exhibit the periodic orbits with different frequencies and the periodic coexistence in the different regions.

To this end, suppose $K(\omega)=0$ has two positive roots $\omega_{1}$ and $\omega_{2}$, where $\omega_{1}>\omega_{2}>0$. Then two sequences of critical values of delay are given by

$$
\tau_{1}^{j}=\left(\varphi_{1}+2 j \pi\right) / 2 \omega_{1}, \quad j=0,1,2, \ldots,
$$


where $\varphi_{1} \in[0,2 \pi)$ satisfies

$$
\cos \varphi_{1}=k_{1}\left(\omega_{1}\right) / k_{3}\left(\omega_{1}\right), \quad \sin \varphi_{1}=k_{2}\left(\omega_{1}\right) / k_{3}\left(\omega_{1}\right),
$$

and

$$
\tau_{2}^{j}=\left(\varphi_{2}+2 j \pi\right) / 2 \omega_{2}, j=0,1,2, \ldots,
$$

where $\varphi_{2} \in[0,2 \pi)$ satisfies

$$
\cos \varphi_{2}=k_{1}\left(\omega_{2}\right) / k_{3}\left(\omega_{2}\right), \quad \sin \varphi_{2}=k_{2}\left(\omega_{2}\right) / k_{3}\left(\omega_{2}\right) .
$$

Moreover, the transversality condition of Hopf bifurcation can be exhibited by substituting $\omega_{1}$ and $\omega_{2}$ into Eq. (4.16) for the critical delayed values $\tau_{1}^{j}$ and $\tau_{2}^{j}, j=0,1,2, \ldots$. However, due to the high order of Eq. (4.13), the detailed expression of positive roots cannot be obtained, which is dependent on the values of system parameters $a_{12}, a_{21}$, and $\gamma$. But it can be easily computed numerically. So, in the following section, we choose the system parameters as $a_{12}=1, a_{21}=-3$, and $\gamma=1$ and have two positive roots $\omega_{1}$ and $\omega_{2}$ in Eq. (4.13). The critical value of delay $\tau_{\mathrm{s}}$ for the fixed system parameters can be obtained by (5.1) and (5.2) numerically. Similar results can be illustrated for different values of system parameters when Eq. (4.13) has two positive roots $\omega_{1}$ and $\omega_{2}$.

For example, choosing $\tau_{1}=0.01$, one has the first and second frequencies, where $\omega_{1}=1.5051$ and $\omega_{2}=1.9921$. Using Eqs. (5.1) and (5.2), two sequences of critical values, i.e., $\tau_{1}^{j}$ and $\tau_{2}^{j}$, are obtained, that is, $\tau_{1}^{j}=0.1520,4.3269,8.5017, \ldots$ and $\tau_{2}^{j}=$ $3.0218,6.1758,9.3298, \ldots$. The dynamic behavior near the trivial equilibrium exhibits a periodic orbit by the Hopf bifurcation if $\tau_{\mathrm{s}} \in(0,0.1520)$. The time history of the periodic orbit is illustrated in Fig. 9(a) with $\tau_{\mathrm{s}}=0.1$. With delay increasing and crossing the critical value $\tau_{1}^{0}=0.1520$, the trivial equilibrium retrieves its stability by the reverse Hopf bifurcation. The system exhibits a stable equilibrium, as shown in Fig. 9(b) for $\tau_{\mathrm{s}}=1$. Moreover, the dynamics of the trivial equilibrium loses its stability when crossing the second critical value $\tau_{2}^{0}=3.0218$. The time history is illustrated in Fig. 9(c) for the fixed delay $\tau_{\mathrm{s}}=3.5$. Then the trivial equilibrium will recover its stability, as shown in Fig. 9(d) with $\tau_{\mathrm{s}}=5$. In such a way, the dynamic behavior near the trivial equilibrium multi-switches its stability when the delay is varied. It is called the dynamical stability switching.

For each fixed delay $\tau_{1}$, using Eqs. (5.1) and (5.2), the critical values of $\tau_{\mathrm{s}}$, i.e., $\tau_{1}^{j}$ and $\tau_{2}^{j}$, $j=0,1,2, \ldots$, are obtained, as shown in Fig. 10 for the system parameters $a_{12}=1, a_{21}=-3$, and $\gamma=1$. That is, we obtain the Hopf bifurcation curve. It divides the $\left(\tau_{1}, \tau_{\mathrm{s}}\right)$-plane into several different regions, where the trivial equilibrium is stable/unstable. So, the dynamic behavior of the trivial equilibrium exhibits the delay-dependent and delay-independent stability. Further, the Hopf bifurcation curves obtained from $\tau_{1}^{1}$ and $\tau_{2}^{1}$ have the first intersection point labeled as $\left(\tau_{1}^{*}, \tau_{\mathrm{s}}^{*}\right)=(0.0451,5.215)$, where the maximum real part eigenvalues are two pairs of pure imaginary values with $\omega_{1}=1.2915$ and $\omega_{2}=2.2934$. This implies that the intersection point is a Hopf-Hopf bifurcation. It follows from the bifurcation theory that the Hopf-Hopf bifurcation will induce the coexistence of two periodic orbits with different frequencies in some parameter regions. The dynamical classification near the Hopf-Hopf bifurcation point is shown in Fig. 11(a). The detailed parameter regions 


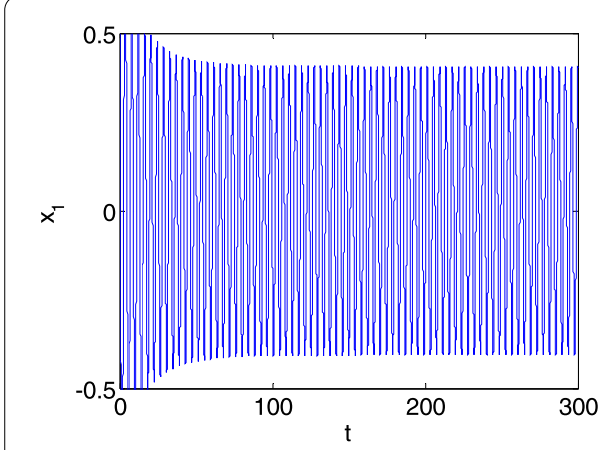

(a)

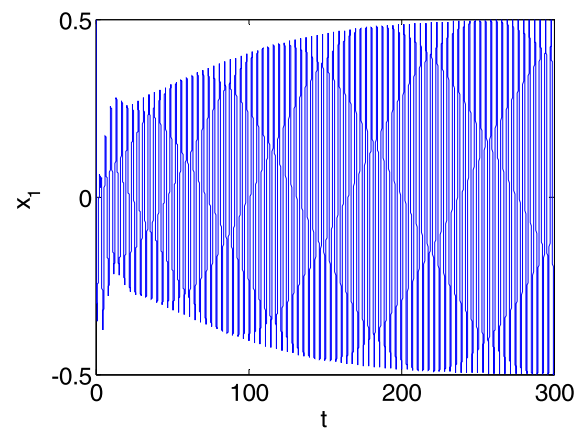

(c)

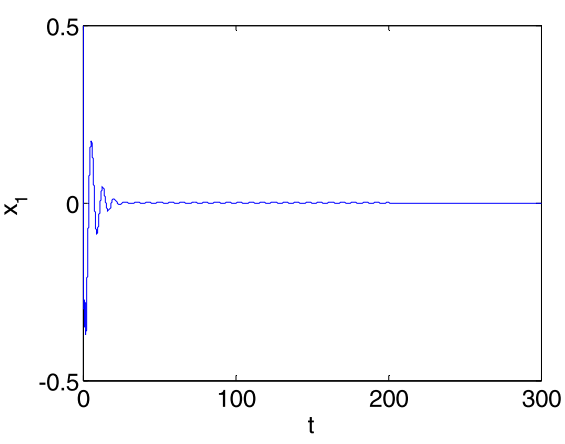

(b)

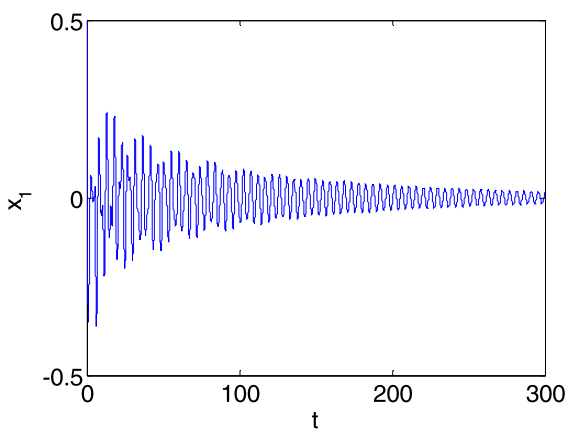

(d)

Figure 9 Time histories with increasing delay $\tau_{\mathrm{s}}$ for (a) $\tau_{\mathrm{s}}=0.1$, (b) $\tau_{\mathrm{s}}=1$, (c) $\tau_{\mathrm{s}}=3.5$, and (d) $\tau_{\mathrm{s}}=5$ and the fixed parameters $a_{12}=1, a_{21}=-3, \gamma=1$, as well as $\tau_{1}=0.01$

Figure 10 Hopf bifurcation curves in $\left(\tau_{1}, \tau_{\mathrm{s}}\right)$-plane illustrates the Hopf-Hopf bifurcation points (labeled as $\mathrm{HH}$ ) for the fixed parameters $a_{12}=1, a_{21}=-3$, and $\gamma=1$, where $\mathrm{S}$ means stable equilibrium

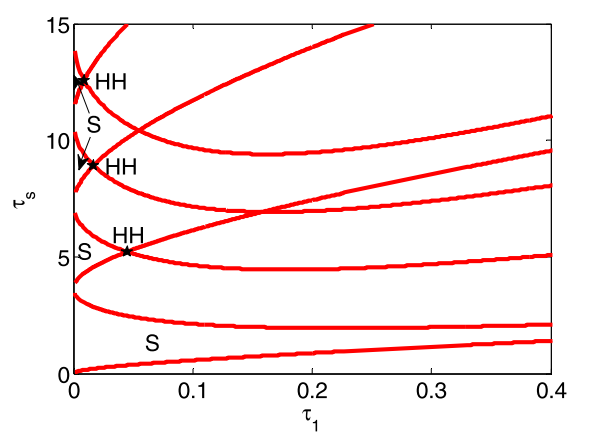

near these bifurcation points can be theoretically presented by the Hopf-Hopf bifurcation normal form. It is a complicated but standard method, which can be applied step by step. In this section, to make it easy to understand, we avoid the boring derivation step and illustrate some numerical simulations to verify the dynamical behaviors. Some Hopf-Hopf bifurcation points and the corresponding frequencies are shown in Table 1.

As in the section above, for the fixed parameters $a_{12}=1, a_{21}=-3$, and $\gamma=1$, the first Hopf-Hopf bifurcation point is $\left(\tau_{1}^{*}, \tau_{\mathrm{s}}^{*}\right)=(0.0451,5.215)$ which is enlarged in Fig. 11(b). System delayed-parameters are chosen to illustrate the different phase portraits near the Hopf-Hopf bifurcation point. The numerical simulations agree well with the dynamical classification. Firstly, we choose the delay $\tau_{1}$ as 0.02 . The system dynamics are illustrated 
Table 1 The first few points of the Hopf-Hopf bifurcation and the roots of (4.13)

\begin{tabular}{lll}
\hline$\left(\tau_{1}^{*}, \tau_{\mathrm{s}}^{*}\right)$ & $\omega_{1}$ & $\omega_{2}$ \\
\hline$(0.0451,5.215)$ & 1.2915 & 2.2934 \\
$(0.017,8.9124)$ & 1.6342 & 1.8357 \\
$(0.00899,12.5791)$ & 1.516 & 1.978 \\
\hline
\end{tabular}

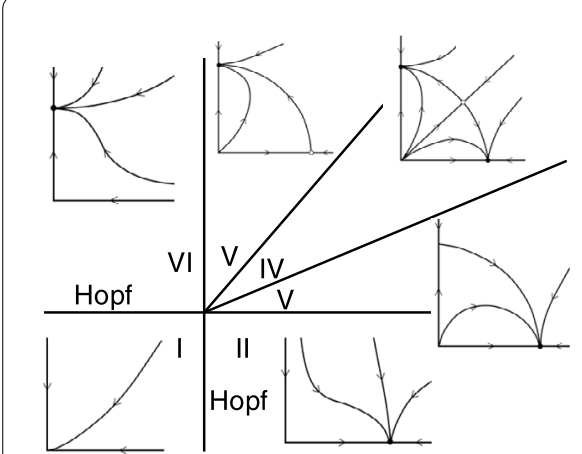

(a)

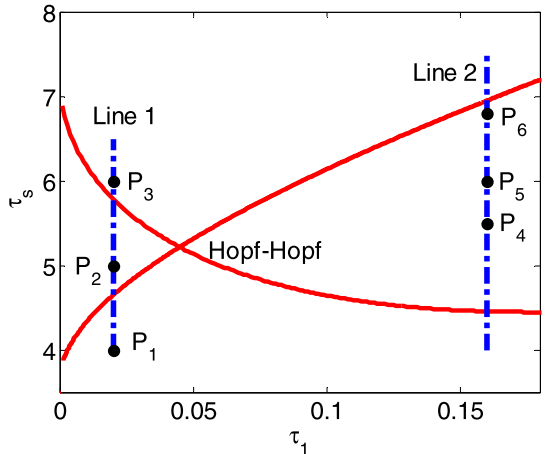

(b)

Figure 11 (a) Classification and bifurcation sets of the Hopf-Hopf bifurcation, (b) several fixed parameter values near the first Hopf-Hopf bifurcation point for numerical simulations

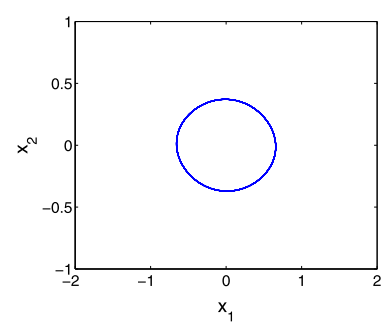

(a)

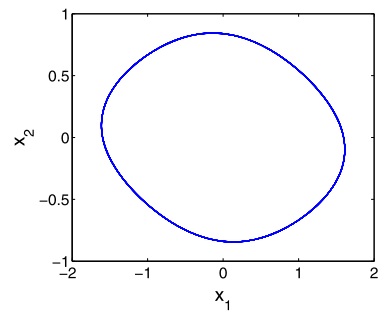

(d)

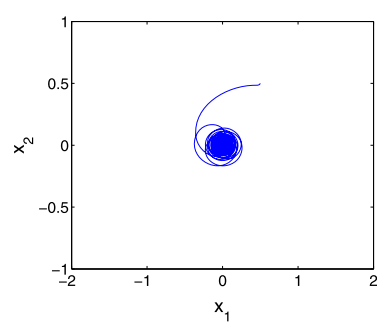

(b)

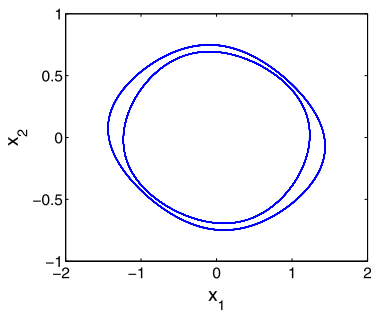

(e)

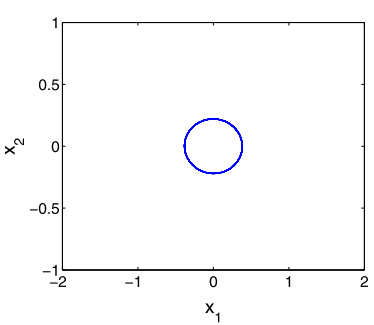

(c)

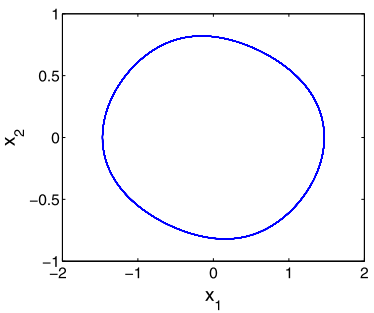

(f)

Figure 12 Phase portraits near the Hopf-Hopf bifurcation point $\left(\tau_{1}^{*}, \tau_{\mathrm{s}}^{*}\right)=(0.0451,5.215)$ for the delay $\left(\tau_{1}, \tau_{\mathrm{s}}\right)$ chosen as (a) $(0.02,4),(\mathbf{b})(0.02,5),(\mathbf{c})(0.02,6),(\mathbf{d})(0.16,5.5),(\mathbf{e})(0.16,6)$, and $(\mathbf{f})(0.16,6.9)$ with the fixed parameters $k_{1}=1, k_{2}=0.6, c_{1}=0.8$

for the different delay $\tau_{\mathrm{s}}=4,5,6$, as shown in Figs. 12(a)-(c). The periodic orbit with the first frequency $\omega_{1}$ (Fig. 12(a)) will evolve into the stable trivial equilibrium (Fig. 12(b)) by the reverse Hopf bifurcation. If the delay crosses through the second Hopf bifurcation 


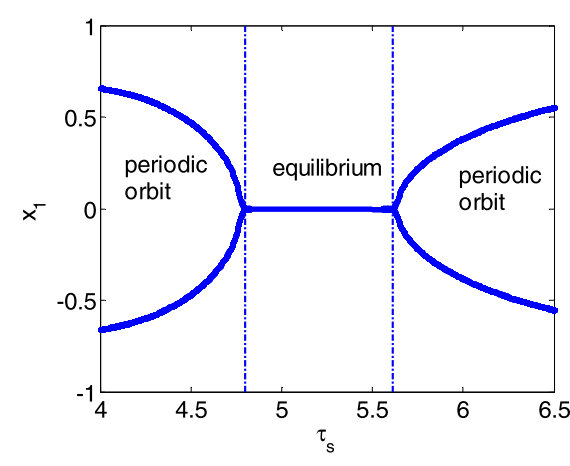

(a)

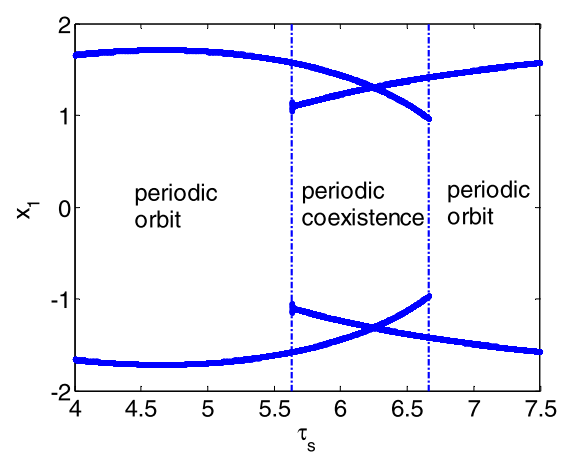

(b)

Figure 13 The one-dimensional bifurcation diagrams show (a) stability switching along Line 1 and (b) periodic coexistence along Line 2 with $a_{12}=1, a_{21}=-3$, and $\gamma=1$

curve, the stable trivial equilibrium loses its stability and enters into the other stable periodic orbit, as shown in Fig. 12(c).

On the other hand, we focus on the complex region and choose the delay parameters labeled as $\mathrm{P}_{4}, \mathrm{P}_{5}$, and $\mathrm{P}_{6}$, as shown in Fig. 11(b). The neural system exhibits periodic coexistence due to the different frequencies. In fact, the periodic orbit with frequency $\omega_{1}$ will be maintained when the delay crosses through the Hopf bifurcation curve, as shown in Fig. $12(\mathrm{~d})$ for $\left(\tau_{1}, \tau_{\mathrm{s}}\right)=(0.16,5.5)$. Further, when the delay parameters are fixed as $\left(\tau_{1}, \tau_{\mathrm{s}}\right)=(0.16,6.9)$, the neural system exhibits the periodic orbit with another frequency $\omega_{2}$, as shown in Fig. 12(f). However, in the evolution process from one periodic orbit to another, the system exhibits the coexistence with two different periodic orbits having different frequencies. The phase portraits are shown in Fig. 12(e) for the different initial values. The delay parameters are fixed as $\left(\tau_{1}, \tau_{\mathrm{s}}\right)=(0.16,6)$ labeled as $\mathrm{P}_{5}$ in Fig. 11(b). In short, numerical simulations are in good agreement with the dynamic classification and bifurcation sets near the Hopf-Hopf bifurcation point. The one-parameter bifurcation diagrams with delay varying are shown in Fig.13.

\section{Conclusion}

Time delay in nonlinear systems has been recognized as an important factor affecting various dynamics [34, 35]. Multistability is one of the most interesting properties in dynamic systems. The long-term behaviors of system trajectories with multistability are easily influenced by the external perturbations. A slight change of a system parameter will induce system dynamics switching from one steady-state to another [36, 37]. In this paper, we illustrated a multi-equilibria coexistence in a delayed Hopfield two-neural system with monotonic activation function. The results have shown that the neural system has two pitchfork bifurcations of the trivial equilibrium. Further, analyzing the characteristic equation of the nontrivial equilibrium generated by the pitchfork bifurcation of the trivial equilibrium, we illustrated the saddle-node bifurcation of the nontrivial equilibria. The system exhibits the multi-coexistences of the stable and unstable equilibria employing the multistage static bifurcations. By the static bifurcation curves of the trivial and nontrivial equilibria, we presented the detailed regions having different numbers of system equilibria in the parameter plane. Then, we proposed the stability analysis and exhibited the periodic orbit using the Hopf bifurcation. The stability regions have been shown by the Hopf 
bifurcation curves. The dynamic behaviors near the Hopf-Hopf bifurcation points have been presented. The system has exhibited the coexistence of the periodic orbits with the different frequencies near the bifurcation points.

Funding

This research is supported by the National Natural Science Foundation of China under Grant No. 11672177, 11672185, and 11472160

\section{Competing interests}

The authors declare that they have no competing interests.

Authors' contributions

All authors read and approved the final manuscript.

\section{Author details}

${ }^{1}$ College of Information Technology, Shanghai Ocean University, Shanghai, China. ${ }^{2}$ College of Marine Science, Shanghai Ocean University, Shanghai, China. ${ }^{3}$ School of Environment and Architecture, University of Shanghai for Science and Technology, Shanghai, China.

\section{Publisher's Note}

Springer Nature remains neutral with regard to jurisdictional claims in published maps and institutional affiliations.

Received: 17 October 2018 Accepted: 18 March 2019 Published online: 03 May 2019

\section{References}

1. Hopfield, J.: Neurons with graded response have collective computational properties like those of two-state neurons. Proc. Natl. Acad. Sci. USA 81, 3088-3092 (1984)

2. Qiu, H., Chen, X., Liu, W., Zhou, G., Wang, Y., Lai, J.: A fast $\ell$ 1-solver and its applications to robust face recognition. J. Ind. Manag. Optim. 8, 163-178 (2012)

3. Wang, Y.J., Zhou, G.L., Caccetta, L., Liu, W.Q.: An alternative Lagrange-dual based algorithm for sparse signa reconstruction. IEEE Trans. Signal Process. 59, 1895-1901 (2011)

4. Guo, Y.X.: Exponential stability analysis of traveling waves solutions for nonlinear delayed cellular neural networks. Dyn. Syst. 32, 490-503 (2017)

5. Guo, Y.X.: Globally robust stability analysis for stochastic Cohen-Grossberg neural networks with impulse control and time-varying delays. Ukr. Math. J. 69, 1220-1233 (2018)

6. Liu, C., Peng, Y.J.: Stability of periodic steady-state solutions to a non-isentropic Euler-Maxwell system. Z. Angew. Math. Phys. 68, 105 (2017)

7. Song, Z.G., Xu, J.: Bifurcation and chaos analysis for a delayed two-neural network with a variation slope ratio in the activation function. Int. J. Bifurc. Chaos 22, 1250105 (2012)

8. Song, Z.G., Xu, J., Zhen, B.: Multi-type activity coexistence in an inertial two-neuron system with multiple delays. Int. J. Bifurc. Chaos 25, 1530040 (2015)

9. Liu, J., Zhao, Z.Q.: Multiple solutions for impulsive problems with non-autonomous perturbations. Appl. Math. Lett. 64, 143-149 (2017)

10. Hao, X., Liu, L.S.: Multiple monotone positive solutions for higher order differential equations with integral boundary conditions. Bound. Value Probl. 2014, 74 (2014)

11. Liu, L.S., Sun, F.L., Zhang, X.G., Wu, Y.H.: Bifurcation analysis for a singular differential system with two parameters via to degree theory. Nonlinear Anal., Model. Control 22, 31-50 (2017)

12. Liu, P., Zeng, Z., Wang, J.: Complete stability of delayed recurrent neural networks with Gaussian activation functions. Neural Netw. 85, 21-32 (2017)

13. Nie, X., Zheng, W., Cao, J.: Coexistence and local $\mu$-stability of multiple equilibrium points for memristive neural networks with nonmonotonic piecewise linear activation functions and unbounded time-varying delays. Neural Netw. 84, 172-180 (2016)

14. Zhang, F., Zeng, Z:: Multistability of recurrent neural networks with time-varying delays and nonincreasing activation function. Neurocomputing 216, 135-142 (2016)

15. Folli, V., Leonetti, M., Ruocco, G.: On the maximum storage capacity of the Hopfield model. Front. Comput. Neurosci. $10,00144(2017)$

16. Rocchi, J., Saad, D., Tantari, D.: High storage capacity in the Hopfield model with auto-interactions-stability analysis J. Phys. A, Math. Theor. 50, 465001 (2017)

17. Kaslik, E., Balint, S.T.: Bifurcation analysis for a discrete-time Hopfield neural network of two neurons with two delays and self-connections. Chaos Solitons Fractals 39, 83-91 (2009)

18. Monteiro, L.H.A., Pellizari Filho, A., Chaui-Berlinck, J.G., Piqueira, J.R.C.: Oscillation death in a two-neuron network with delay in a self-connection. J. Biol. Syst. 15, 49-61 (2007)

19. Gupta, P.D., Majee, N.C., Roy, A.B.: Stability, bifurcation and global existence of a Hopf-bifurcating periodic solution for a class of three-neuron delayed network models. Nonlinear Anal. 67, 2934-2954 (2007)

20. He, X., Li, C., Shu, Y.: Bogdanov-Takens bifurcation in a single inertial neuron model with delay. Neurocomputing 89 193-201 (2012)

21. Song, Z.G., Xu, J.: Stability switches and Bogdanov-Takens bifurcation in an inertial two-neuron coupling system with multiple delays. Sci. China, Technol. Sci. 57, 893-904 (2014) 
22. Dong, T., Liao, X.F., Huang, T.W.: Hopf-Pitchfork bifurcation in an inertial two-neuron system with time delay. Neurocomputing 97, 223-232 (2012)

23. Song, Z.G., Wang, C.H., Zhen, B.: Codimension-two bifurcation and multistability coexistence in an inertial two-neuron system with multiple delays. Nonlinear Dyn. 85, 2099-2113 (2016)

24. Ge, J.H., Xu, J.: Weak resonant double Hopf bifurcations in an inertial four-neuron model with time delay. Int. J. Neural Syst. 22, 63-75 (2012)

25. Song, Z.G., Xu, J.: Stability switches and double Hopf bifurcation in a two-neural network system with multiple delays. Cogn. Neurodyn. 7, 505-521 (2013)

26. Song, Z.G., Xu, J.: Stability switches and multistability coexistence in a delay-coupled neural oscillators system. J. Theor. Biol. 313, 98-114 (2012)

27. Guan, Y.L., Zhao, Z.Q., Lin, X.L.: On the existence of positive solutions and negative solutions of singular fractional differential equations via global bifurcation techniques. Bound. Value Probl. 2016, 141 (2016)

28. Mao, X.C.: Bifurcation, synchronization, and multistability of two interacting networks with multiple time delays. Int. J. Bifurc. Chaos 26, 1650156 (2016)

29. Ge, J.H., Xu, J. Li, Z.Q.: Zero-Hopf bifurcation and multistability coexistence on a four-neuron network model with multiple delays. Nonlinear Dyn. 87, 2357-2366 (2017)

30. Song, Z.G., Yang, K., XU, J., Wei, Y.C.: Multiple pitchfork bifurcations and multiperiodicity coexistences in a delay-coupled neural oscillator system with inhibitory-to-inhibitory connection. Commun. Nonlinear Sci. Numer. Simul. 29, 327-345 (2015)

31. Liz, E., Ruiz-Herrera, A.: Attractivity, multistability, and bifurcation in delayed Hopfield's model with non-monotonic feedback. J. Differ. Equ. 255, 4244-4266 (2013)

32. $\mathrm{Ma}$, J.F., Wu, J.H.: Multistability and gluing bifurcation to butterflies in coupled networks with non-monotonic feedback. Nonlinearity 22, 1383-1412 (2009)

33. Morita, M.: Associative memory with non-monotone dynamics. Neural Netw. 6, 115-126 (1993)

34. Sun, X., Perc, M., Kurths, J.: Effects of partial time delays on phase synchronization in Watts-Strogatz small-world neuronal networks. Chaos 27, 053113 (2017)

35. Sun, X., Perc, M., Kurths, J., Lu, Q.: Fast regular firings induced by intra- and inter-time delays in two clustered neuronal networks. Chaos 28, 106310 (2018)

36. Hao, X., Zuo, M., Liu, L.: Multiple positive solutions for a system of impulsive integral boundary value problems with sign-changing nonlinearities. Appl. Math. Lett. 82, 24-31 (2018)

37. Wang, B., Wu, X., Meng, F: Trigonometric collocation methods based on Lagrange basis polynomials for multi-frequency oscillatory second order differential equations. J. Comput. Appl. Math. 313, 185-201 (2017)

\section{Submit your manuscript to a SpringerOpen ${ }^{\circ}$ journal and benefit from:}

- Convenient online submission

- Rigorous peer review

- Open access: articles freely available online

- High visibility within the field

Retaining the copyright to your article

Submit your next manuscript at $\gg$ springeropen.com 